\title{
Low Probability of Interception Beampattern Using Frequency Diverse Array Antenna
}

\author{
Shaddrack Yaw Nusenu* \\ ${ }^{1}$ Koforidua Technical University, Electrical / Electronic Department, Faculty of Engineering, Ghana. \\ A R T I C L E I N F O \\ Article history: \\ Received: 12 February, 2017 \\ Accepted: 31 March, 2017 \\ Online: 02 April, 2017 \\ Keywords: \\ Frequency diverse array \\ costa sequence \\ Low probability of intercept \\ Transmit beamforming

\begin{abstract}
A B S T R A C T
Frequency diverse array (FDA) antenna employs a small frequency increment, as compared with the carrier frequency, across antenna array elements. The use of a frequency increment generates an array factor that is dependent on angle, time, and the range and this yields a range and angle dependent beampattern. In this paper, a transmit array beamforming FDA employing costa sequence frequency increments is proposed that offers low probability of interception (LPI) for radar systems. Because active radars are highly visible to intercept receivers, FDA beam with uniform linear array and non-linearly increasing frequency offsets to reduce the system visibility is replaced by the conventional high gain phased-array antenna beam. The proposed method produce random-like peak distribution without obvious peaks making it difficult to be detected by unfriendly detector or intercept receivers without known of the specified coding sequence for the frequency increments. Numerical simulation results verify the proposed method.
\end{abstract}

\section{Introduction}

Phased-array antenna has been extensively employed in many applications such as radar, electronic warfare, radio astronomy and airport safety etc. This is because it can steer the high-gain beam electronically with high effectiveness towards any desired direction [1, 2, 3]. The directional gain obtainable by employing phased-array antenna is beneficial for detecting, tracking weak targets and suppressing sidelobe interferences from other directions [4]. Conversely, the high gain beam is visible to intercept receivers which will degrade the capability of the surveillance system [5]. Furthermore, active surveillance radars are highly susceptible to detection and interception by opposing forces. The electronic support measures (ESM) can be used to intercept the radar transmission and possibly facilitate harmful electronic counter-measures (ECM) techniques [6]. Therefore it is essential to develop low probability of intercept (LPI) radar systems.

LPI radar system employs a distinct emitted waveform intended to prevent a non-cooperative intercept receiver from intercepting and detecting its emission. On the other hand, low probability of identification (LPID) radar system uses a special radiated waveform intended to prevent a non-cooperative intercept receiver from intercepting and detecting its emission but if intercepted, identification of the radiated waveform modulation and its parameters becomes difficult. It is worth mentioning that LPID radar is an LPI radar [7].

Several techniques have been introduced to reduce radar visibility and improve its LPI ability [8]. Mostly, three main areas are considered in order to reduce radar visibility [6]: (i) spreading the energy in time with high duty cycle waveforms (ii) spreading the energy in frequency with wide bandwidth waveforms (iii) spreading the energy in space through broad transmitter antenna beams. In [9], frequency hopping, orthogonal frequency division multiplexing (OFDM), and random waveforms have been put forward for LPI radars. In [10], switched antenna technique called antenna hopping method which uses irregular scan patterns to reduce the susceptibility to receivers was introduced to enhance the LPI performance. Although with specific beamforming technique, the probability of being detected in sidelobe region can be reduced. In [6], LPI transmit array beamforming method was proposed which employed phased array antenna. Even though phased-array antenna has been widely employed for numerous applications, it produce fixed steering beam at an angle for all the ranges [11].

A new flexible antenna array called frequency diverse array (FDA) has been introduced in [12, 13]. The authors reported that the range-angle-time dependent far-field ar-

* Shaddrack Yaw Nusenu, Koforidua Technical University, Ghana, Email: nusenu2012gh@yahoo.com 
ray pattern formation are the core attributes of the concept. FDA was defined in [15] as an antenna aperture where the frequency of each antenna element is controlled independently to steer the main beam, depending on the time domain of each frequency component in the far-field. The most significant feature of FDA, as opposed to phasedarray, is that the former employs a small frequency increment across its antenna array elements. This frequency increment makes the array beampattern to change as a function of the range, angle and time [11, 12, 13]. In [14], FDA radar using costas sequence modulated frequency increments was proposed to decoupled the range and angle beampattern, in which only FDA applications are considered.

In this paper, LPI FDA transmit beamforming with costa sequence frequency increments is proposed. The proposed method transmitted beampattern will have a randomlike peak distribution without obvious peaks. This implies that it will be difficult for the intercept receiver from intercepting and detecting without known of the specified coding sequence for the frequency increments.

The rest of the paper is organized as follows: Section 2 proposes the LPI beamforming using costas sequence FDA antenna, which is verified in Section 3 with numerical results. Finally Section 4 concludes the paper.

\section{LPI FDA BEAMFORMING}

Consider an FDA with $M$ elements which can be excited by either the same waveform or different waveforms. In this paper, we assume costas sequence frequency increments used for the $m$ th element is $\Delta f_{m}$ as shown in Figure 1. In the costas coding technique frequencies for the subpulses are chosen in a random pattern, according to some predetermined rule. Consider the frequency-time matrix shown in Figure 2(a), (b) and (c). In this case, the rows are indexed from $i=1,2, \ldots, M$ and the columns are indexed from $j=0,1,2, \ldots,(M-1)$. The rows and columns are used to denote subpulses and distinct frequencies, respectively. As shown in Figure 2(a), (b) and (c) a dot indicates the distinct frequency value assigned to the associated subpulse. In this fashion, the frequency assignments are chosen randomly. The element of the difference matrix in row $i$ and column $j$ is given as

$$
D_{i, j}=a_{i+j}-a_{j}, i+j \leq M
$$

where $a_{i}$ is the $i$ th element of the coding sequence. The remaining locations (where $i+j>M$ ) are left blank. Using (1) the first row is formed by taking differences between adjacent elements in the coding sequence, then second row by taking differences between next-adjacent elements, and so forth. We employ this technique for FDA antenna, thus, only one carrier frequency is transmitted by any one of the $M$ FDA elements and each carrier frequency is permitted only once. In [16], the details for the construction algorithms for costas signals can be found.

The radiated frequency fed to the $m$ th of the LPI FDA using costas frequency increments can be given as

$$
f_{m}=f_{0}+\Delta f_{m}, m=0,1,2, \ldots, M-1
$$

where $f_{0}$ and $\Delta f_{m}$ are the carrier frequency and frequency increment, respectively, and $M$ is the number of array elements. Specifically, standard FDA is achieved when $\Delta f_{m}=\mathrm{m} \Delta f$. Suppose a far-field position with range and angle $(r, \theta)$, the phase of the transmitted signal of $m$ th element is represented by

$$
\begin{gathered}
\alpha_{m}(r, \theta)=\left(f_{0}+\Delta f_{m}\right) r_{m} \beta \\
r_{m}=r+m d \sin \theta, m=0,1,2, \ldots, M-1
\end{gathered}
$$

where $d$ is the element spacing. Hence, the phase difference between the signals transmitted by the $m$ th and first elements can be approximately given as

$$
\psi_{m}(r, \theta)=-\beta f_{0} m d \sin \theta+\Delta f_{m} r \beta-\beta m \Delta f_{m} d \sin \theta
$$

Next, we apply a set of costas frequency coding, $\Delta f_{m}$ and phases, $\delta_{m}(r)$ to each element to achieve a "spoiled' low-gain beamforming. Hence the transmit array factor (AF), which can be seen as the fundamental basis pattern [17, 18] is expressed as

$A F_{0}(r, \theta)=\sum_{m=0}^{M-1} e^{-j \beta f_{0} m d \sin \theta} \cdot e^{j \Delta f_{m} \beta r} \cdot e^{-j \beta \Delta f_{m} m d \sin \theta} \cdot e^{-j \delta_{m}(r)}$

where $\Delta f_{m}$ is designed using Welch-constructed costas coding sequence and $\delta_{m}(r)$ can be designed using optimization algorithms. By applying a linear phase progression to (6) in increments of $\kappa=\frac{2 \pi}{N}$ for first pattern, $2 \kappa$ for the second pattern and so forth, additional patterns can be created. In such a fashion, $(N-1)$ patterns can be generated. Therefore (6) can be formulated as (7) [18] at the top of the next page, where $n=1,2, \ldots, N$.

It is worth mentioning that, these $N$ steered versions of the fundamental guarantee linear independence. Moreover, they all exhibit low gain and broad beamwidth. Therefore their peak power can be significantly reduced in any directions to ensure LPI property.

Furthermore, to retain the array radar performance and simultaneously achieving LPI property, we synthesize the high gain patterns along the desired direction by linear combinations of the basis patterns. Suppose the $n$th basis pattern is weighted by the complex coefficient $w_{0, n}$, then $N$ transmit beampatterns can be expressed in matrix formulation as given in (8), [6, 18].

This implies that using (8), high gain beampatterns can be formed by linearly combinations of the $N$ basis patterns.

\section{NUMERICAL RESULTS}

In the simulations, we assume the LPI FDA operating at a carrier frequency of $f_{0}=10 \mathrm{GHz}$. An 32 elements uniform linear array is used for the standard FDA and phased-array beamforming (see Figure 4). The transmit array element spacing is designed to be $d=\frac{c_{0}}{2 f_{0}}$. We consider the Welch constructed costas coding sequence for $M=10$, with the chosen positions as $1,2,4,8,5,10,9,7,3,6$. Similarly, for $M$ $=12$, we have $1,2,4,8,3,6,12,11,9,5,10,7$ and $M=15$, as $2,8,9,12,4,14,10,15,13,7,6,3,11,1,5$. These costas sequence coding with $M=10, M=12, M=15$, respectively, was used for LPI FDA transmit beamforming for the arrays and 


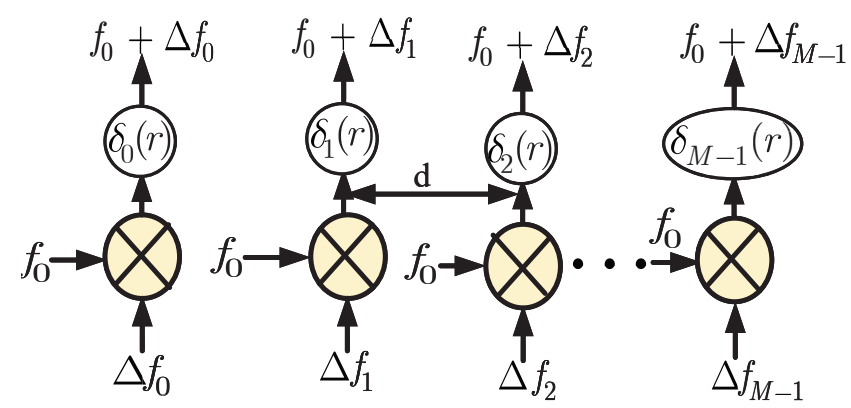

Figure 1: Illustration of LPI FDA. $\delta_{m}$ and $\Delta f_{m}$ denotes phase shifters and costas coding frequency increments for the $m$ th element, repectively.

$$
A F_{N-1}(r, \theta)=\sum_{m=0}^{M-1} e^{-(N-1) m \kappa} e^{-j \beta f_{0} m d \sin \theta} \cdot e^{-j \Delta f_{m} \beta r} \cdot e^{-j \beta \Delta f_{m} m d \sin \theta} \cdot e^{-j \delta_{m}(r)}
$$

$$
\left[\begin{array}{c}
A_{0}(r, \theta) \\
\vdots \\
A_{N-1}(r, \theta)
\end{array}\right]=\left[\begin{array}{ccc}
w_{0,0} & \cdots & w_{0, N-1} \\
\vdots & \ddots & \vdots \\
w_{N-1,0} & \cdots & w_{N-1, N-1}
\end{array}\right]\left[\begin{array}{c}
A F_{0}(r, \theta) \\
\vdots \\
A F_{N-1}(r, \theta)
\end{array}\right]
$$

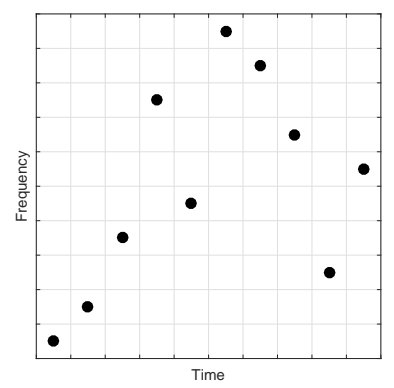

(a)

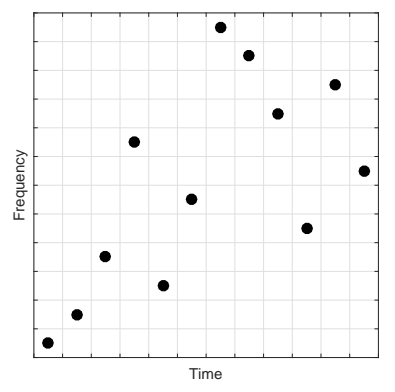

(b)

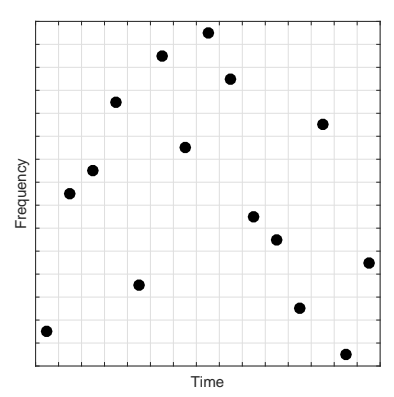

(c)

Figure 2: Frequency-time matrix representations of costas sequence coding: (a) $M=10$, (b) $M=12$, (c) $M=15$. 


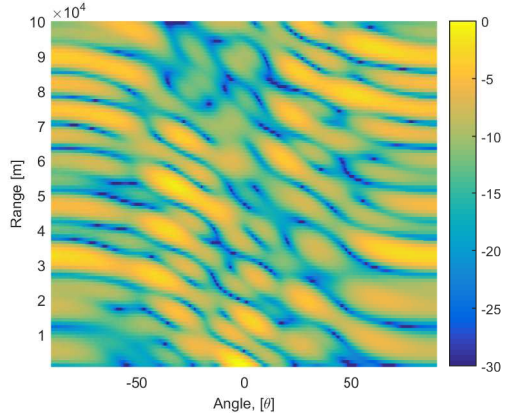

(a)

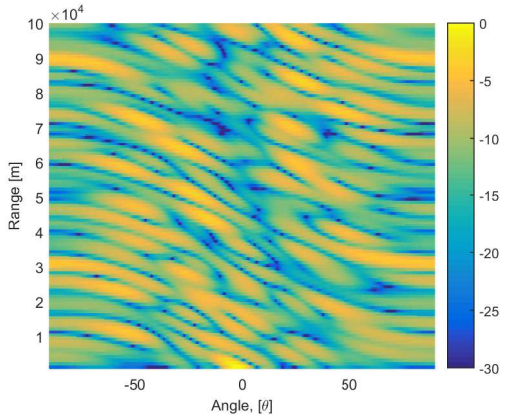

(b)

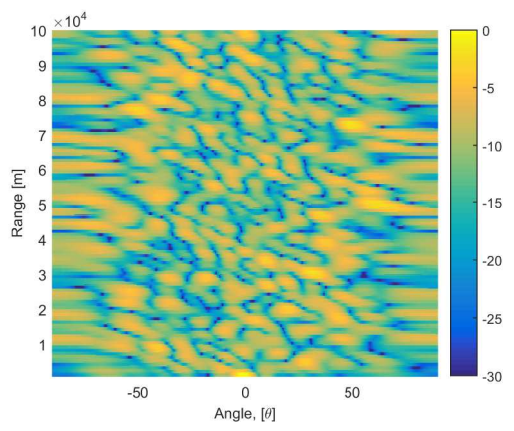

(c)

Figure 3: Illustration of LPI FDA beampattern using costas sequence coding: (a) $M=10$, (b) $M=12$, (c) $M=15$.

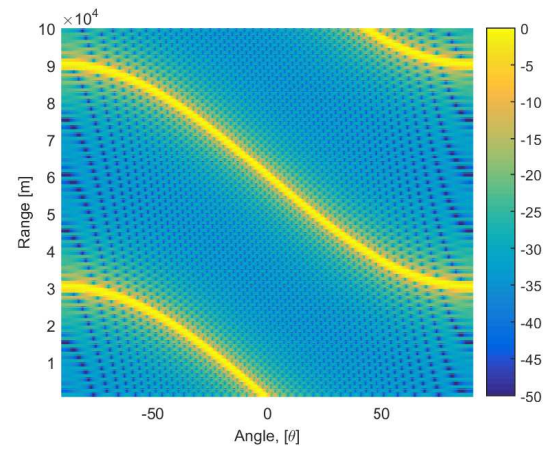

(a)

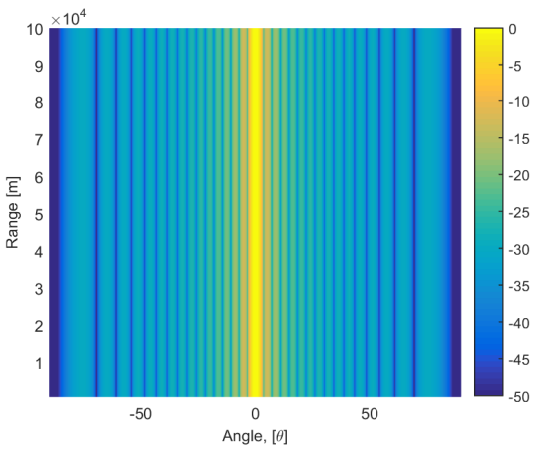

(b)

Figure 4: Illustration of transmit beampattern: a) Standard FDA, b) Phased-array. 


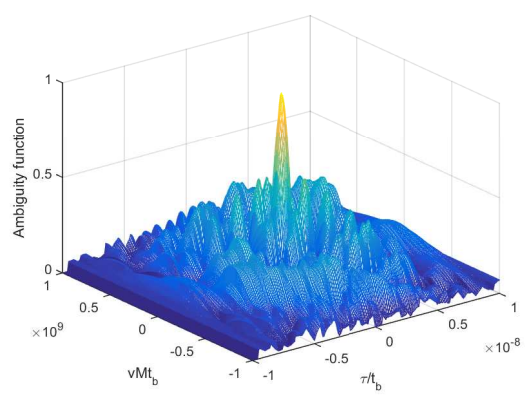

(a)

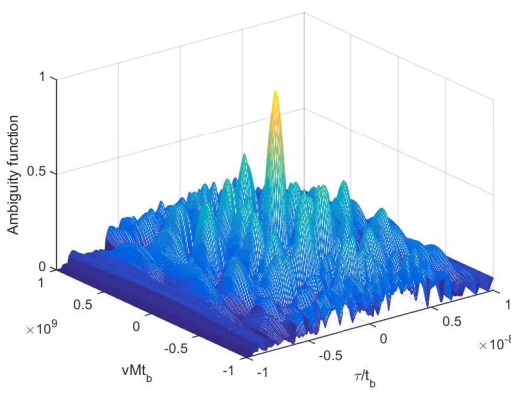

(b)

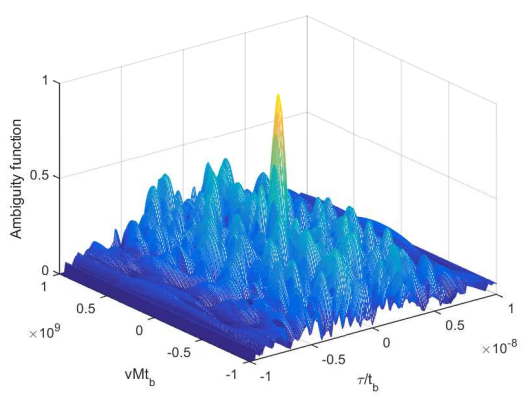

(c)

Figure 5: Illustration of three-dimensional ambiguity function LPI FDA using costas sequence coding: (a) $M=10$, (b) $M$ $=12$, (c) $M=15$.

their frequency-time matrix representation is shown in Figure 2.

Next, we show the LPI FDA transmit beamforming employing costas sequence with $M=10, M=12, M=15$, respectively, in Figure 3. It can be seen that for the respective costas sequence coding employed, the LPI FDA transmit beamforming has random-like peak distributions without noticeable peaks. This characteristics implies that it is very difficult to be intercepted and detected by intercept receivers or unfriendly detectors without the knowledge of the specified costas coding sequence used for the frequency increments.

Suppose $\Delta f=5 \mathrm{kHz}$, Figure 4(a) shows the standard FDA when specifically $\Delta f_{m}=\mathrm{m} \Delta f$. It can be observed that it produces range and angle dependent and range-angle coupling transmit beamforming. This feature can be exploited for range and angle localization of radar targets. Differently, the phased-array produce angle-dependent only transmit beamforming, as shown in Figure 4(b). However, the phased-array provides no range information because of its range-independent beamforming.

Finally, the ambiguity function of LPI FDA costas coding sequence is shown in Figure 5 for distinct $M$. It can be seen that the ambiguity functions accumulates into a spike, thus clearly resembles thumb-tack response.

\section{CONCLUSION}

This paper proposed a transmit beamforming technique using FDA which offers LPI for surveillance systems. The high-gain phased-array antenna beam is replaced with the low gain FDA beams with nonlinear frequency increments to reduce the system visibility. The proposed technique achieves the same performance as the traditional high-gain beam. The obvious advantage of the proposed technique presented here is the random-like peak energy distributions without noticeable peaks. The results show that the proposed technique has the advantages of both FDA radar and guarantee LPI property. The frequency increments are chosen from Welch constructed costas coding sequence across the array element to ensure the LPI property for radar systems. Overall, the proposed technique can possibly be combined with other LPI waveform design techniques to further reduce the susceptibility and visibility of LPI radar systems.

Conflict of Interest The author declare no conflict of interest.

Acknowledgment The author would like to thank Respected Professor Wen-Qin Wang (Senior Member - IEEE) of UESTC for his great contributions, support and encouragement.

\section{References}

[1] T. A. Lam, D. C. Vier, J. A. Nieslsen, C. G. Parazzoli, and M. H. Tanielian. "Steering phased array antenna beams to the horizon using a buckyball NIM lens,'Proc. IEEE, vol. 99, no. 10, pp. 1755-1767, Oct. 2011.

[2] B. Bauman, A. Christianson, A. Wegener, and W. J. Chappell. "Dynamic visualization of antenna pat- 
terns and phased-array beam steering,'IEEE Antennas Propag. Mag., vol. 54, no. 3, pp. 184-193, Jun. 2012.

[3] C. J. Reddy. "Phased array-based systems and applications,'IEEE Antennas Propag. Mag., vol. 39, no. 5, pp. 102-103, Oct. 1997.

[4] Li, J., Stoica, P. "The phased array is the maximum SNR active array,'IEEE Signal Process. Mag., 27, (2), pp. 143-144, 2010.

[5] O. G. Vendik and D. S. Kozlov. "Phased antenna array with a sidelobe cancellation for suppression of jamming,'IEEE Antennas and Wireless Propagation Letters, vol. 11, 2012.

[6] D. E. Lawrence. "Low probability of intercept antenna array beamforming,"IEEE Transactions on Antennas and Propagation, vol. 58, no. 9, pp. 2858-2865, September 2010.

[7] Phillip E. Pace. "Detecting and Classifying Low Probability of Intercept Radar,'Second Edition 2009.

[8] V. Krisnamurthy. "Emission management for low probability intercept sensors in network centric warfare,'IEEE Transactions on Aerospace and Electronic Systems, vol. 41, no. 1, pp. 133-151, January 2005.

[9] W.-Q. Wang. "MIMO SAR OFDM chirp waveform diversity design with random matrix modulation,'IEEE Transactions on Geoscience and Remote Sensing, vol. 53, no. 3, pp. 1615-1625, March 2015.

[10] E. J. Baghdady. "Directional signal modulation by means of switched spaced antennas,'IEEE Transactions on Communications, vol. 38, no. 4, pp. 399-403, April 1990.
[11] W.-Q. Wang. "Frequency diverse array antenna: New opportunites,'IEEE Antennas and Propagation Magazine, vol. 57, no. 2, pp. 145-152, April 2015.

[12] P. Antonik, M. C. Wicks, H. D. Griffiths, and C. J. Baker. "Range dependent beamforming using element level waveform diversity," in Proceedings of the International Waveform Diversity and Design Conference, Las Vegas, USA, pp. 1-4, January 2006.

[13] P. Antonik, M. C. Wicks, H. D. Griffiths, and C. J. Baker. "Frequency diverse array radars," in Proceedings of the IEEE Radar Conference, Verona, NY, April pp. 215-217, 2006.

[14] Shaddrack Yaw Nusenu, Zhe Wang, Wen-Qin Wang. "FDA radar using Costas sequence modulated frequency increments," in Proceedings of CIE International Radar Conference, pp. 1058-1061. 2016.

[15] Wen-Qin Wang, H. C. So, and Alfonso Farina. "An Overview on Time/Frequency Modulated Array Processing ," DOI 10.1109/JSTSP.2016.2627182, IEEE Journal of Selected Topics in Signal Processing.

[16] S. W. Golomb and H. Taylor. "Constructions and properties of costas arrays,"Proceedings of the IEEE, vol. 72, no. 9, pp. 1143-1163, September 1984.

[17] Wen-Qin Wang. "Adaptive Rf Stealth Beamforming For Frequency Diverse Array Radar,"23rd European Signal Processing Conference (EUSIPCO) pp. 11581161, 2015.

[18] Wen-Qin Wang. "Moving-Target Tracking by Cognitive RF Stealth Radar Using Frequency Diverse Array Antenna,'IEEE Transactionson Geoscience And Remotesensing, vol. 54, no. 7, pp. 3764-3773, July 2016. 